\title{
Prostate immunotherapy: should all guns be aimed at the prostate-specific antigen?
}

\section{"...we have several potential antigens that might be useful targets of prostate immunotherapy."}

\section{KEYWORDS: cancer vaccine " immunotherapy $\approx$ prostate cancer}

Prostate cancer continues to be a significant health problem and an economic burden all over the world. It is the second most common cancer in men and the sixth leading cause of cancer-related deaths worldwide [1]. As life expectancy is increasing, there is a possibility for a significant increase in the incidence of prostate cancer, with a corresponding increase in the number of cancer patients. Improved diagnostic utilities have shown that more than $90 \%$ of prostate cancer cases are detected in its localized stage and are effectively manageable. However, when cancer cells spread outside the prostate gland (metastatic), medical treatments begin with androgen ablation and, ultimately, undergo a variety of chemotherapy options. Unfortunately, due to treatment failure, prostate cancer can relapse and become castration-resistant, with an estimated death rate of approximately 258,000 men worldwide [1], directing a need for improved treatment modalities for advanced and metastatic prostate cancer.

Over the last two decades, a surge in immunological manipulations of the host inducing antitumor response has resulted in the development of vaccines targeting tumor-specific antigens, as well as immunotherapeutic drugs. When conventional treatments fail, immunebased therapies have shown improved patient survival, and are now emerging as a vanguard for the treatment of cancer. The blueprint for the success of immunotherapy agents has been demonstrated in multiple clinical trials for prostate cancer and melanoma [2-4]. The immunological targeting of tumor involves tumorassociated antigen(s), cytokines and costimulatory molecules and immune-regulatory agents (CTLA-4 and PD-1), emphasizing the engagement of the immune system-enhancing antitumor activity.

\section{Targeted antigens for prostate immunotherapy}

Prostate cancer provides a unique opportunity for immunotherapy as prostate cancer cells express a number of antigens that serve as potential targets. The best known target antigens include prostatic acid phosphatase (PAP), prostate-specific antigen (PSA), prostate-specific membrane antigen (PSMA) and prostate stem cell antigen (PSCA). Prostate immunotherapy has advanced using antibody and T-cell-based approaches; however, immune reactions against cancer cells are frequently triggered by the stimulation of adaptive immunity via the activation of cytotoxic $\mathrm{CD}^{+}$ $\mathrm{T}$ lymphocytes targeting specific antigens to prostate cancer cells.

Several vaccine approaches targeting castration-resistant prostate cancer (CRPC) are under investigation; however, the dendritic cell (DC) -based vaccine targeting PAP (sipuleucel-T) is the most advanced tumor vaccine approved by the US FDA for the treatment of metastatic CRPC [4]. Sipuleucel-T, serving as a personalized medicine, utilizes autologous DCs with ex vivo loading of PAP and GM-CSF fusion proteins designed to induce a PAP-specific immune response. Although the sipuleucel-T vaccine demonstrated improved overall survival, the anti-PAP CD8 ${ }^{+}$T-cell response were not detectable. Instead, measurement of T-cell responses are broadly confined to T-cell proliferation and its mechanistic function remain a question of discussion [5]. Similarly, DNA-based vaccine targeting of PAP demonstrated its safety profile in a Phase I/IIa clinical study, while other Phase II clinical studies are ongoing [6].

Immunotherapy targeting PSA is one of the most studied approaches that has utilized PSAspecific peptides and mRNA, as well as full length cDNA inducing PSA-specific immune

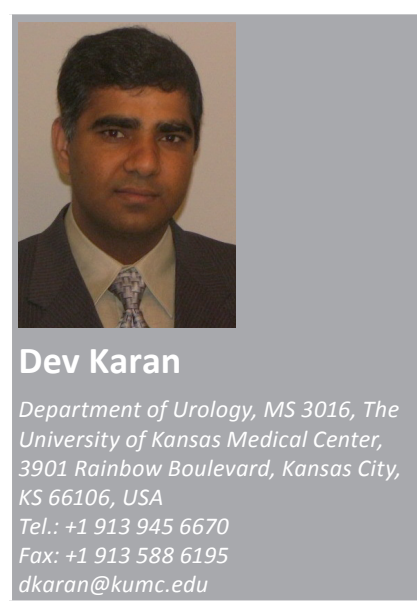

Future $\because: \%$
Medicine ${ }_{\text {part of }}$ 
and antitumor responses. The delivery of PSA as a tumor vaccine includes plasmid DNA, DCs and microbial vectors such as Vaccinia and fowlpox viral vectors (Prostvac-VF ${ }^{\circledR}$; Bavarian Nordic, Kvistgaard, Denmark), adenovirus vector and Listeria monocytogenes (reviewed in [3]). While immunotherapy approaches targeting PSA are at various developmental stages, Prostvac-VF is at the most advanced stage currently in a Phase III clinical trial. This approach uses a prime-boost strategy with Vaccinia (priming) and fowlpox (boosting) viral vectors, each expressing a cassette of PSA and a triad of costimulatory molecules (ICAM-1, B7.1 and LFA-3). In a completed Phase II clinical study, Prostvac-VF revealed an 8.5-month improvement in median survival in men with metastatic CRPC [7]. PSA-targeted immunotherapy using adenovirus type 5 as a vaccine vector is in Phase II clinical trial, while other strategies using Listeria, plasmid DNA, peptide or DCs are at various stages of development [3]. PSA as an immunotherapy target has clearly demonstrated the generation of PSA-specific $\mathrm{CD}^{+} \mathrm{T}$ cells in both preclinical and clinical studies $[8,9]$. In a mouse model, a recent study also described the use of PSA-targeted immunotherapy for simultaneous induction of anti-PSA antibody (IgE) and anti-PSA cytotoxic lymphocyte response, but its clinical applicability needs to be explored [10].

\section{"When conventional treatments fail, immune- based therapies have shown improved patient survival, and are now emerging as a vanguard for the treatment of cancer."}

PSA is the only FDA-approved diagnostic biomarker for prostate cancer routinely used in clinics. PSA is a secretory protein and its presence in the blood above a certain limit indicates the development of abnormal cell growth in the prostate gland, including benign prostatic hyperplasia, prostatitis or prostate cancer. The biochemical recurrence of prostate cancer is primarily diagnosed based on PSA level in the blood; however, there are still a number of prostate cancer patients where PSA levels do not reflect the state of the disease, leading to a debate about the utility of PSA [11]. Although its use as a diagnostic marker has been controversial only recently, the potential to explore the usefulness of PSA as an immunotherapy target is due to the fact that its expression is very much restricted to the epithelial cells of the prostate. It is worth mentioning that the level of PSA in the blood depends on multiple factors [12], and that despite a low PSA level, prostate cancer cells will still be expressing PSA, projecting it as a promising target for immunotherapy.

Similarly, PSMA is also considered a potential immunotherapeutic target due to its nature of expression within the prostate and its increased expression in prostate cancer cells following androgen ablation [13]. In preclinical models, multiple vaccine approaches have utilized PSMA as a target antigen; however, their move into the clinic has been slow. Early studies for PSMAbased immunotherapy were derived using antibody targeting intracellular (7E11) and then later on extracellular (J591) epitopes for the detection and in vivo imaging of its subsequent target on prostate cancer cells [14]. An autologous DC-based vaccination using recombinant PSMA (DCVax prostate; Northwest Biotherapeutics, MD, USA) showed clinical benefit in a Phase I/II clinical study delaying the progression of metastatic diseases; however, its long-term clinical benefit remains to be investigated. A recent Phase I/II study on 32 patients using a DNA-based vaccine targeting PSMA demonstrated the safety profile with a measurable T-cell response and an increase in PSA doubling time [15]. This study revealed that the $\mathrm{CD}^{+} \mathrm{T}$ cell compared with the $\mathrm{CD}^{+} \mathrm{T}$ cell response, were detectable in a higher number of patients.

Prostate immunotherapy is also exploring the use of PSCA as a novel target since it is a membrane-bound protein stably expressed in advanced prostate cancer, and its level increases with the progression and metastasis of the disease $[16,17]$. PSCA has been investigated both as an antibody-mediated and T-cell-mediated immunotherapy target. PSCA directed tumor vaccines using DCs, plasmid DNA and adenovirus in combination with a chaperone or adjuvants are being explored in preclinical studies. Other candidates that are generating interest for prostate immunotherapy include MUC1, NY-ESO-1, six transmembrane epithelial antigen of the prostate and the androgen receptor; however, more studies are needed to support their importance as a potential candidate for the advancement of prostate immunotherapy.

\section{Immune spectrum of prostate antigens}

Based on the nature of antigen, the spectrum of peptides during antigen processing and presentation bound to MHC I and MHC II molecules develop T-cell recognition leading to activation of $\mathrm{CD}^{+}$and $\mathrm{CD} 4^{+} \mathrm{T}$ cells, respectively. 
Although PSMA and PAP are well-established antigens associated with prostate cancer progression, the use of PSMA as a systemic target could be inconvenient due to its involvement in multiple physiological functions [18]. Similarly, specificity of PAP was questioned due to its wide expression in various tissues [19]. Clinical or preclinical studies have not provided any clear evidence that the use of PSMA or PAP antigens as a vaccine dominantly induces antigenic $\mathrm{CD} 8^{+} \mathrm{T}$ cells essential for the killing of tumor targets. Early preclinical studies in a rat model demonstrating the utility of PAP as an immunotherapy target predominantly showed the development of a PAP-specific $\mathrm{CD}^{+}{ }^{+} \mathrm{T}$-cell response. A Phase I/IIa study of DNA-based vaccine had evidence of a $\mathrm{CD} 8^{+} \mathrm{T}$-cell response in two out of $22(9 \%)$ patients, while a $\mathrm{CD}^{+}$ T-cell response were detectable in nine out of $22(\sim 41 \%)$ patients [6]. Since the role of Tregs is well accepted in hindering the effect of antitumor immunity, use of a tumor-associated antigen inducing a higher number of $\mathrm{CD}^{+} \mathrm{T}$ cells compared with $\mathrm{CD} 8^{+} \mathrm{T}$ cells may not be beneficial. Such an additional increase in the number of $\mathrm{CD}^{+} \mathrm{T}$ cells due to immunization would possibily contribute to the pool of $\mathrm{CD}^{+}$Tregs supporting immune suppression by tumor cells. We have also observed, in our preclinical studies, that the use of a full-length antigen inducing antigenic $\mathrm{CD}^{+} \mathrm{T}$ cells outweigh the benefit of antigens that primarily induce $\mathrm{CD} 8^{+} \mathrm{T}$ cells to inhibit the growth of established prostate tumors. Therefore, the use of antigens such as PSMA and PAP as an immunotherapy target for prostate cancer may need careful consideration with additional combination of immunotherapy drugs. In an effort to induce a broad spectrum of tumor-specific immunity, prostate GVax $^{\circledR}$ (Aduro BioTech, CA, USA) immunotherapy used GM-CSF-modified irradiated

\section{References}

1 Jemal A, Bray F, Center MM, Ferlay J, Ward E, Forman D. Global cancer statistics. CA Cancer J. Clin. 61, 69-90 (2011).

2 Pardoll DM. Immunology beats cancer: a blueprint for successful translation. Nat. Immunol. 13, 1129-1132 (2012).

3 Karan D, Holzbeierlein JM, Van Veldhuizen P, Thrasher JB. Cancer immunotherapy: a paradigm shift for prostate cancer treatment. Nat. Rev. Urol. 9, 376-385 (2012).

4 Kantoff PW, Higano CS, Shore ND et al. Investigators. Sipuleucel-T immunotherapy

tumor cell lines (LNCaP and PC3) as the tumor vaccine. While expected to strengthen the antitumor immunity due to the development of a wide range of immune and antitumor responses, the prostate GVax was prematurely terminated in Phase III clinical studies due to safety concerns [20].

On the other hand, the major advantage of PSA as an immunotherapy target has been its high specificity to the prostate and the development of $\mathrm{CD}^{+} \mathrm{T}$ cells dependent on killing of prostate tumor cells. Based on multiple clinical studies, it appears that prostate immunotherapy targeting PSA has provided more consistent and somewhat satisfactory results compared with other antigens. It is noticeable that a combined use of PSA and PSCA antigens prevail CD8 ${ }^{+}$ T-cell response and may prove better targets to improve the efficacy of prostate immunotherapy [9]. Slowly but surely, we have several potential antigens that might be useful targets of prostate immunotherapy. Keeping in view the heterogeneity of prostate cancer, identification of other biomarkers would be valuable for a combined use in vaccination to increase the likelihood of targeted tumor antigens by the cytotoxic $T$ cells for improved prostate immunotherapy.

\section{Acknowledgements}

Thanks to JB Thrasher, Chairman, Department of Urology, for his critical reading of this article.

\section{Financial \& competing interests disclosure}

Research in the author's laboratory is supported by the NIH (R21CA169453). The author has no other relevant affiliations or financial involvement with any organization or entity with a financial interest in or financial conflict with the subject matter or materials discussed in the manuscript apart from those disclosed.

No writing assistance was utilized in the production of this manuscript.

for castration-resistant prostate cancer. N. Engl. J. Med. 363, 411-422 (2010).

5 Sheikh NA, Petrylak D, Kantoff PW et al. Sipuleucel-T immune parameters correlate with survival: an analysis of the randomized Phase 3 clinical trials in men with castrationresistant prostate cancer. Cancer Immunol. Immunother. 62, 137-147 (2013).

6 McNeel DG, Dunphy EJ, Davies JG et al. Safety and immunological efficacy of a DNA vaccine encoding prostatic acid phosphatase in patients with stage D0 prostate cancer. J. Clin. Oncol. 27, 4047-4054 (2009).

7 Kantoff PW, Schuetz TJ, Blumenstein BA et al. Overall survival analysis of a Phase II randomized controlled trial of a poxviralbased PSA-targeted immunotherapy in metastatic castration-resistant prostate cancer. J. Clin. Oncol. 28, 1099-1105 (2010).

8 Lubaroff DM, Konety BR, Link B et al. Phase I clinical trial of an adenovirus/ prostate-specific antigen vaccine for prostate cancer: safety and immunologic results. Clin. Cancer Res. 15, 7375-7380 (2009).

9 Karan D, Dubey S, van Veldhuizen P, Holzbeierlein JM, Tawfik O, Thrasher JB. Dual antigen target-based immunotherapy for prostate cancer eliminates the growth of established tumors in mice. Immunotherapy 3, 735-746 (2011). 
10 Daniels-Wells TR, Helguera G, Leuchter RK et al. A novel IgE antibody targeting the prostate-specific antigen as a potential prostate cancer therapy. BMC Cancer 13, 195 (2013).

11 McNaughton-Collins MF, Barry MJ. One man at a time - resolving the PSA controversy. N. Engl. J. Med. 365, 1951-1953 (2011).

12 Lilja H, Ulmert D, Vickers AJ. Prostatespecific antigen and prostate cancer: prediction, detection and monitoring. Nat. Rev. Cancer 8, 268-278 (2008).

13 Wright GL, Jr., Grob BM, Haley C et al. Upregulation of prostate-specific membrane antigen after androgen-deprivation therapy. Urology 48, 326-334 (1996).
14 Akhtar NH, Pail O, Saran A, Tyrell L, Tagawa ST. Prostate-specific membrane antigen-based therapeutics. Adv. Urol. 973820 (2012).

15 Chudley L, McCann K, Mander A et al. DNA fusion-gene vaccination in patients with prostate cancer induces high-frequency CD8(+) T-cell responses and increases PSA doubling time. Cancer Immunol. Immunother. 61, 2161-2170 (2012).

16 Dannull J, Diener PA, Prikler L et al. Prostate stem cell antigen is a promising candidate for immunotherapy of advanced prostate cancer. Cancer Res. 60, 5522-5528 (2000).

17 Reiter RE, Gu Z, Watabe T, Thomas G et al. Prostate stem cell antigen: a cell surface marker overexpressed in prostate cancer. Proc. Natl Acad. Sci. USA 95, 1735-1740 (1998).

18 Rajasekaran AK, Anilkumar G, Christiansen JJ. Is prostate-specific membrane antigen a multifunctional protein? Am J. Physiol. Cell Physiol. 288, C975-C981 (2005).

19 Quintero IB, Araujo CL, Pulkka AE et al. Prostatic acid phosphatase is not a prostate specific target. Cancer Res. 67, 6549-6554 (2007).

20 Le DT, Pardoll DM, Jaffee EM. Cellular vaccine approaches. Cancer J. 16, 304-310 (2010). 